\title{
Management of Leakage into the Abdomen due to the Involvement of Jejunum in the Incision Line
}

\author{
Seracettin Egin
}

\begin{abstract}
Here, we present the management of a patient, developing severe peritonitis due to laceration of the jejunum, involving sutures applied to facial layers after anterior resection for adenocarcinoma of the sigmoid colon. The patient, an 83-year woman, was operated by anterior resection, and relaparotomy was performed because the small bowel contents leaked from the incision. A compulsory stoma on the top of the incision was performed. Bilateral fasciocutaneous advancement flaps were carried out to treat the patient with severe intraabdominal sepsis via vacuum-assisted closure. The patient, with Apache-II score 12, open abdomen Bjorck score 2C, and Mannheim peritonitis index score 28, was monitored in the intensive care unit. Based on our experience, open abdomen management with vacuum-assisted closure might be successful for patients with intraabdominal sepsis because of uncontrollable primer sources. We propose that the technique defined for this patient is an innovative technique for primary source control of open abdomen patients.
\end{abstract}

Key Words: Open abdomen, Vacuum-assisted closure, Intraabdominal sepsis.

\section{INTRODUCTION}

Continuous enteral leakage into the abdomen is the primary source of severe sepsis in the abdomen. The control of the primary source for the treatment of severe sepsis is mandatory. Recent studies have pointed out that mortality rates of abdominal sepsis dramatically increase in the event of severe sepsis and septic shock. Severe sepsis is described as sepsis associated with at least one acute organ dysfunction, hypoperfusion or hypotension. ${ }^{1}$ Several laparotomies might be required in case of continuing sepsis. An open abdomen (OA) has significant advantages, including the ability to perform subsequent laparotomies more efficiently and prevent the onset of abdominal compartment syndrome (ACS). In patients with severe sepsis and septic shock, OA allows an easy second-look to control the source of infection and evacuate inflamed and toxic contents, reducing the load of peritoneal cytokines and other inflammatory substances, and preventing their production by removing the source itself. ${ }^{2}$

Here, we present the management of a patient, developing severe peritonitis due to laceration of the jejunum, involving sutures applied to facial layers after anterior resection for adenocarcinoma of the sigmoid colon.

\section{CASE REPORT}

An 83-year woman underwent anterior resection for adenocarcinoma of the sigmoid colon. Ten days later, a

Department of Surgery, University of Health Sciences,

Okmeydani Education and Research Hospital, Istanbul, Turkey

Correspondence: Dr. Seracettin Egin, Department of Surgery,

University of Health Sciences, Okmeydani Education and

Research Hospital, Istanbul, Turkey

E-mail: seracettin_egin@hotmail.com

Received: July 10, 2018; Accepted: October 20, 2018 relaparotomy had to be carried out due to leakage of contents from the incision site. A full layer suture cutting was identified on the jejunum. The abdomen under the incision was contaminated by small bowel contents. Because of the intra-abdominal adhesions, the distance of the jejunum cutting to the Treitz ligament was not presently measurable. Since the interrupted jejunum was not sufficiently dissected due to adhesions, the edge of the jejunum cutting was fixed to the stoma on the top of the incision. After 10 days, the small bowel contents leaked under the incision line and took over the management of the patient at this stage.

The inside of the abdomen was dirty with the contents of the small intestine leaking from the side of the stoma (Figure 1A). An intraabdominal fluid culture was taken, and the dirty abdomen was cleaned with $6 \mathrm{~L}$ of warm saline solution. To achieve an OA approach, it was necessary that the edge of the stoma was completely isolated from the inside of the abdomen for primer source control and that management was performed using a vacuum-assisted closure (VAC) system. For this purpose, bilateral fasciocutaneous advancement flaps were prepared (Figure 1B). The flaps were joined in the midline by stitching together, and the edge of the stoma was sutured to the joined flaps (Figure 1C). OA was temporarily closed with VAC. This procedure was carried out using the Abthera open abdomen management system, which is produced by $\mathrm{KCl}$ Licensing Inc. (Acelity) in Athlone, Dublin. An Abthera system has one central sponge and six additional sponge extensions. All of the sponges are covered with visceral organ protective layers. Six additional sponge extensions were embedded completely into the intra-abdominal cavity after washing of the abdomen with saline. An elliptical uncovered sponge was applied above six additional sponge extensions. The back face of the elliptical uncovered sponge was at the fascia level, and its front face was at the level of the skin. The elliptical uncovered 


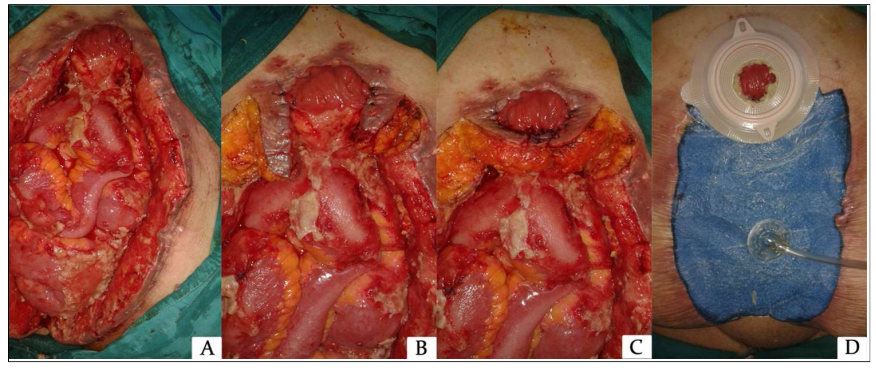

Figure 1: (A) The inside of the abdomen contaminated with the contents of the small intestine leaking from the side of the stoma. (B) Bilateral fasciocutaneous advancement flaps. (C) The flaps that joined on the midline by stitching together and the stoma that was sutured to joined flaps. (D) The VAC system and a colostomy adapter that was applied over the skin around the stoma.

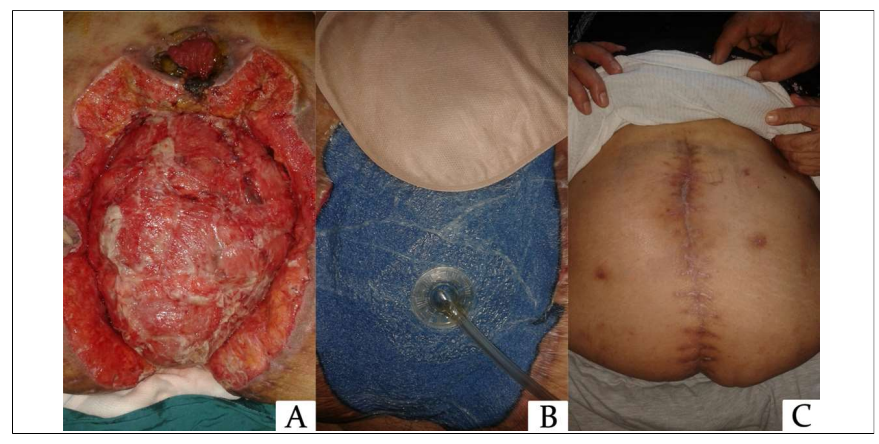

Figure 2: (A) The clean abdomen after 6 days of VAC therapy. (B) A colostomy adapter and bag that were again applied over the skin around the stoma. (C) On the day of discharge from the hospital.

sponge and skin of the abdomen were covered with sterile and adhesive drapes. This was connected with $125 \mathrm{mmHg}$ negative pressure to vacuum devices via a collecting system, and negative pressure therapy (NPT) was started smoothly. A colostomy adapter was applied over the skin around the stoma (Figure 1D). The patient was monitored in the intensive care unit. The Apache-II score was 12, open abdomen Bjorck score was $2 \mathrm{C}$, and Mannheim peritonitis index score was 28. Enteral nutrition was terminated, and total parenteral nutrition (TPN) was started. Escherichia coli was isolated from the intraabdominal fluid culture. After 6 days, VAC exchange was performed in the operation room by washing of the abdomen with $3 \mathrm{~L}$ of warm saline solution. The clean abdomen during this procedure is shown in Figure 2A. A colostomy adapter and bag were again applied over the skin around the stoma (Figure 2B). No bacteria were isolated from the intraabdominal fluid culture at this stage. The VAC was removed on the 11th day of the VAC application. The stoma was closed with side to side anastomosis. The subcutaneous layer in both sides of the incision was dissected from the fascia, and the skin was closed. No additional problems developed during the intensive care unit and surgery clinic stay. The patient was discharged one and half month after the primary surgery (Figure 2C).

\section{DISCUSSION}

VAC techniques have been extensively used in patients with OA for temporary abdominal wall closure. OA with
VAC is associated with significantly improved survival in abdominal sepsis requiring laparotomy. ${ }^{3}$ The abdominal contents are potentially exposed and thus must be protected with a temporary coverage. ${ }^{4}$ Studies made in recent years show that the $\mathrm{OA}$ technique with the temporary abdominal wall closure using negative pressure dressing methods gives positive results. ${ }^{5-7}$ Advances in the management techniques of $\mathrm{OA}$ and new NPT devices have significantly reduced the previously observed prohibitive morbidity associated with OA.8 If an early fascial closure is impossible, commercially prepared negative pressure dressings are available. These dressings can be changed under general anesthesia with intervals of 2-4 days for subsequent dressings. NPT actively discharges toxins and bacteria-rich intraperitoneal fluid. The advantages of the VAC system include prevention of ACS and loss of abdominal domain, collection and monitoring of fluid loss, no damage to the skin or abdominal fascia. Disadvantages of this approach include the need for general anesthesia to change the VAC system and expensive commercial equipment.

In conclusion, the functional VAC system requires a sturdy skin to fixate the adhesive drapes. In these patients, the sturdy skin around stoma might be achieved with bilateral fasciocutaneous advancement flaps. OA management with VAC systems might be successful for patients with intraabdominal sepsis because of uncontrollable primer sources. We propose that the technique defined for this patient is an innovative technique for primary source control of OA patients.

\section{REFERENCES}

1. Sartelli M, Catena F, Di Saverio S, Ansaloni L, Malangoni M, Moore EM, et al. Current concept of abdominal sepsis: WSES position paper. World J Emerg Surg 2014; 9:22.

2. Sartelli M, Abu-Zidan FM, Ansaloni L, Bala M, Beltrán MA, Biffl WL, et al. The role of the open abdomen procedure in managing severe abdominal sepsis: WSES position paper. World J Emerg Surg 2015; 10:35.

3. Bleszynski MS, Chan T, Buczkowski AK. Open abdomen with negative pressure device $v s$. primary abdominal closure for the management of surgical abdominal sepsis: A retrospective review. Am J Surg 2016; 211:926-32.

4. Coccolini F, Montori G, Ceresoli M, Catena F, Moore EE, Ivatury $\mathrm{R}$, et al. The role of open abdomen in non-trauma patient: WSES Consensus Paper. World J Emerg Surg 2017; 12:39.

5. Kaplan M, Banwell P, Orgill DP, Ivatury RR, Demetriades $D$, Moore FA, et al. Guidelines for the management of the open abdomen. Wounds 2005; 17 (1 Suppl):1-24.

6. Campbell A, Chang M, Fabian T, Franz M, Kaplan M, Moore F, et al. Management of the open abdomen: From initial operation to definitive closure. Am Surg 2009; 75(11 Suppl):S1-22.

7. Demetriades D. Total management of the open abdomen. Int Wound J 2012; 9 (Suppl 1):17-24.

8. Leppäniemi A, Kimball EJ, De Laet I, Malbrain ML, Balogh ZJ, De Waele JJ. Management of abdominal sepsis - A paradigm shift? Anaesthesiol Intensive Ther 2015; 47:400-8. 